\title{
Stamping System with Automated Feeding Based on Programmable Logic Controller Electronic Cam Function
}

\author{
Chien-Yu Lu, ${ }^{1}$ Wen-Yi Houng, ${ }^{1}$ Chun-Wan Chang, ${ }^{1}$ \\ Sen-Hu Yen, ${ }^{1}$ Chia-Liang Tseng, ${ }^{1}$ and Te-Jen $\mathrm{Su}^{2}, 3^{*}$ \\ ${ }^{1}$ Department of Industrial Education and Technology, National Changhua University of Education, \\ Changhua City 500, Taiwan, ROC \\ $2^{2 *}$ Department of Electronic Engineering, National Kaohsiung University of Science and Technology, \\ Kaohsiung City 807618, Taiwan, ROC \\ ${ }^{3}$ Graduate Institute of Clinical Medicine, Kaohsiung Medical University, \\ Kaohsiung City 80708, Taiwan, ROC
}

(Received June 20, 2021; accepted September 1, 2021; online published December 21, 2021)

Keywords: feeding manipulator, programmable controller, cam curve, synchronous control

Products in the stamping industry have been widely used in 3C products (computers, communications, and consumer electronics), daily necessities, and transportation vehicles. With the rapid development of the manufacturing industry and the rising cost of traditional methods of production, labor, equipment, and materials, it is essential to invest in automated production equipment. Therefore, improvements in stamping equipment with automated feeding to increase production capacity have been valued and adopted by enterprises. Manufacturers have also invested in the development and production of stamping equipment. The main purpose of this study is to improve the stamping process to reduce labor and increase production capacity. First, a 3D feeding manipulator is simulated. Its control system adopts a programmable logic controller with a motion control module, which is then connected to a servo unit via Mechatrolink-III onsite network communication. An electronic cam synchronous tracking function is used to control the trajectory between the manipulator and the punch and reduce the punch's waiting time for the displacement of the manual parts of the feeding machine, thereby increasing the production efficiency. The control system is matched with a human-machine interface, which makes it easier to operate, test the mold, set the cam curve, and troubleshoot. Thus, product quality and production capacity can be significantly improved.

\section{Introduction}

The purpose of this paper is to explore the use of a programmable logic controller (PLC) via motion control (MC) with a module's electronic cam (ECAM) synchronization function, which is designed for the feeding of a hand-controlled stamping equipment system, and a humanmachine interface (HMI) to study the motion trajectory.

Molds are known as the mother of industry, and most of the products and parts in industry need to be stamped and processed using molds. ${ }^{(1)}$ Owing to the high production efficiency,

${ }^{*}$ Corresponding author: e-mail: sutj@nkust.edu.tw

https://doi.org/10.18494/SAM.2022.3478 
consistent quality, high rigidity, high material utilization, recyclability, and low cost of mold machining, it currently cannot be replaced by other machining methods. ${ }^{(2)}$ Stamping products are widely used in food, clothing, housing, daily necessities, hardware parts, 3C products (computer, communications, and consumer electronics), bicycle parts, automobile and motorcycle sheet metal parts, and vehicles. Therefore, stamping is a pivotal process in production.

With the globalization of trading and market competition, the manufacturing industry is undergoing a large-scale digital transformation. Industry 4.0, the fourth industrial revolution, has arrived. The government of the Republic of China (Taiwan) advocates digital transformation and in-depth AI research and development. For example, in the traditional stamping process, loading and unloading are mostly carried out manually owing to equipment technology and cost restrictions. However, repetitive loading and unloading can easily cause fatigue and distraction, leading to reduced physical and mental health, productivity, and precision. ${ }^{(3)}$ Thus, manual feeding no longer meets production requirements. Automated feeding mechanisms have become indispensable for stamping. Stamping peripheral equipment will vary depending on the production process. The combination of commonly used automated stamping production lines, such as those shown in Figs. 1 and $2,{ }^{(4)}$ and increased industrial automation have emerged as pressing issues for enterprises.

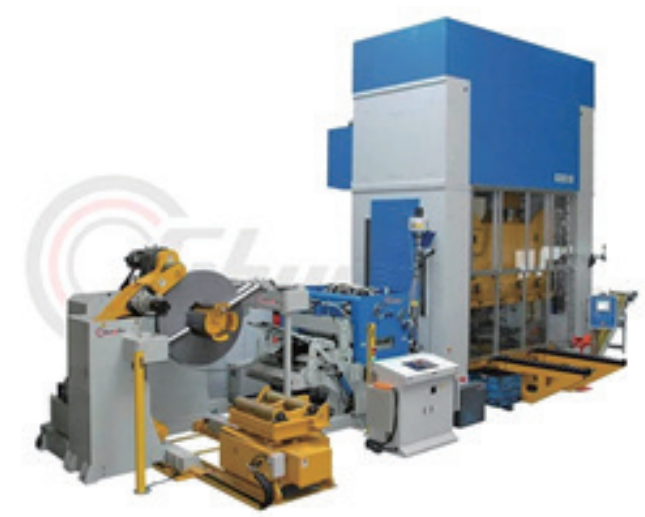

Fig. 1. (Color online) Stamping three-in-one automated computer numerical control (CNC) feeder. ${ }^{(4)}$

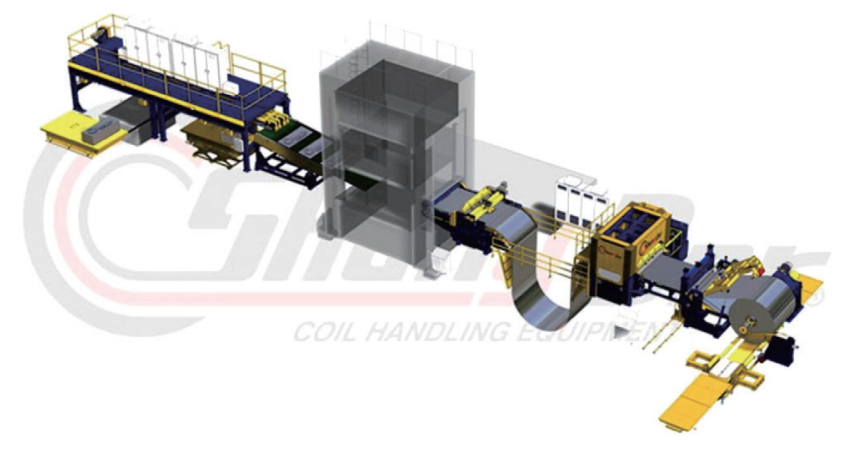

Fig. 2. (Color online) Sheet metal stamping and blanking line. ${ }^{(4)}$ 
This research was motivated by the desire of customers to reduce labor and increase production capacity by improving the stamping production line process. We consider a company producing a product that requires five stamping processes, five punching tools, and five operators working $8 \mathrm{~h}$ per day with a maximum production capacity of about 2500 to 3000 pieces. The production capacity is unable to meet the order requirements, thus causing delays in delivery as well as loss of orders. In addition, owing to the manual repetitive loading and unloading and stamping noise, there is a risk to the physical and mental health of the operators, which may increase the probability and severity of work safety incidents. To improve the stamping process, three solutions are proposed. The first solution is to equip each press with a set of automated conveying equipment and industrial manipulators. For this solution, the labor requirement is low, but the investment cost is very high and the increase in production capacity is limited. The second solution is to equip each punch with a set of vibrating barrel automated feeding equipment and an automated loading and unloading system. ${ }^{(5)}$ For this solution, the labor requirement is low and the investment cost is low, but the productivity is limited and the product is easily damaged. The third solution is to propose a 200 ton punch press and a multistation 3D feeding manipulator, plus a two-in-one leveler and a numerically controlled (NC) left and right offset feeder. This reduces the requirement for labor and greatly increases the production capacity, although the investment cost is high. After evaluation, the client adopts the third solution.

The 3D feeding manipulator is important equipment that dominates the production capacity of the entire stamping production line, and the motion trajectory control between the feeding manipulator and the punching action is the focus of this study. ${ }^{(6)}$ Motion trajectory control is achieved by the synchronous tracking control of the electronic cam. ${ }^{(7)}$ Many workpieces in stamping production require multiple stamping processes to obtain the required shape and size. The traditional stamping production mode has the disadvantages of high cost, low efficiency, low positioning accuracy, and safety concerns. The use of multistation automated feeding manipulator technology can eliminate these problems. ${ }^{(8)}$

According to the literature, ${ }^{(9)}$ a multistation punching machine production line installed to replace a punching line with 5-6 punching machines has a work efficiency 4-5 times that of a manual feeding line and 2-3 times that of an automated single-machine production line. Equipment investment can be reduced by $20-40 \%$, energy consumption can be reduced by 50 $70 \%$, and the total cost of stamping parts can be reduced by $40-70 \%$. The production space, the number of operators, and investment in equipment can be reduced, and the safety of the production line can be increased. The requirements for the mass production of products are thus fulfilled. A production line with multistation automated stamping is mainly composed of a feeding system, a punching machine, a 3D feeder system, and a conveying and unloading system, as shown in Fig. 3. The 3D feeder is responsible for moving stamping parts between stations. The feeding modes mainly include mechanical feeding and electronic feeding. ${ }^{(10)}$

Owing to their higher production efficiency, multistation press production lines are favored by automobile manufacturers in Europe, the United States, Japan, South Korea, and other countries to maximize benefits and meet the needs of large-scale automobile manufacturing. The distance between the stations of multistation stamping machines should be increased to 


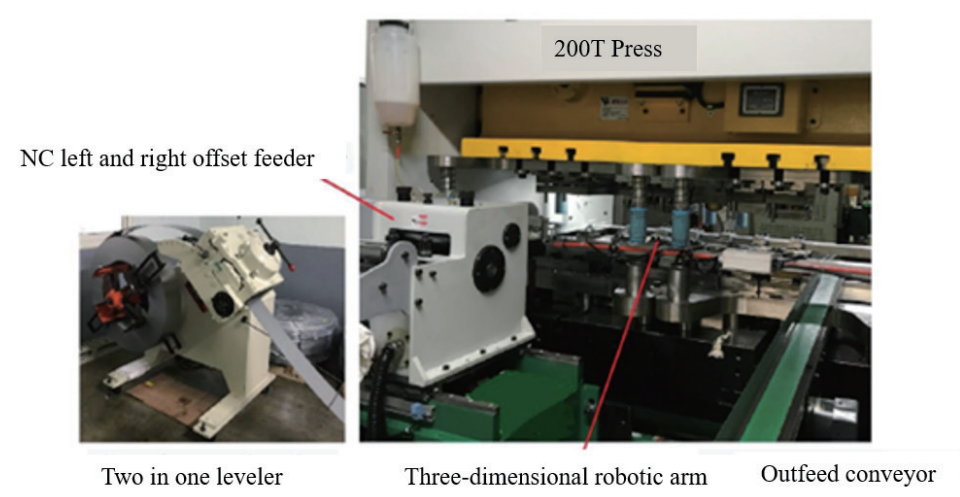

Fig. 3. (Color online) Schematic diagram of multistation stamping production line.

accommodate the need to stamp very large metal sheets for vehicles. ${ }^{(10)}$ Synchronous control refers to the function of using the synchronization parameter setting configuration of each axis to synchronize the position of the slave axis with respect to the position of the synchronized master axis. The position of the synchronous slave axis relative to the synchronous master axis is calculated from the set cam (curve) data, gear setting, clutch setting, compensation setting, and other data. For the synchronous spindle, the command and current coordinates of each axis including the counter input value or virtual axis can be selected. ${ }^{(11)}$

Generally, the mechanical cam component is composed of three parts: the original mover, the frame, and the follower. The frame mainly provides support, as shown in Fig. 4. In the application process, the original moving and driven parts must coordinate with each other. Starting from the rotation or reciprocating movement of the original moving part, the driven part is gradually driven to move, and the complex mechanical movement is completed. In addition, with increasing usage time of the mechanical cam, its wear increases and the amount of noise produced increases. ${ }^{(12)}$

An electronic CAM (ECAM) is essentially an intelligent controller that uses analog cam motion curves for mechanical control. The ECAM can be adjusted according to the input, making it different from a mechanical cam, which has the disadvantages of easy wear, poor accuracy, and a complicated design. The ECAM has the advantages of lower cost and time, and the capability of setting the intensity according to requirements. A reasonable cam curve can make the cam movement softer and more flexible. ${ }^{(12)}$ In automated production or logistics conveying lines, reciprocating actions such as workpiece grasping and placing are common. A cam-type pick-and-place manipulator has the advantages of a simple, compact structure and high reliability. ${ }^{(12,13)}$ A variety of designs have been proposed for cam pick-and-place manipulators. For example, a double-chambered manipulator, which can complete the output action of "lift-forward-descent-backward" has been proposed. ${ }^{(14)}$ The mechanism improves the output accuracy of the follower and has a compact structure. A small double-cylindrical cam manipulator that can very effectively follow a predetermined work trajectory with a stable speed and a rapid response has also been proposed. ${ }^{(15)}$ 


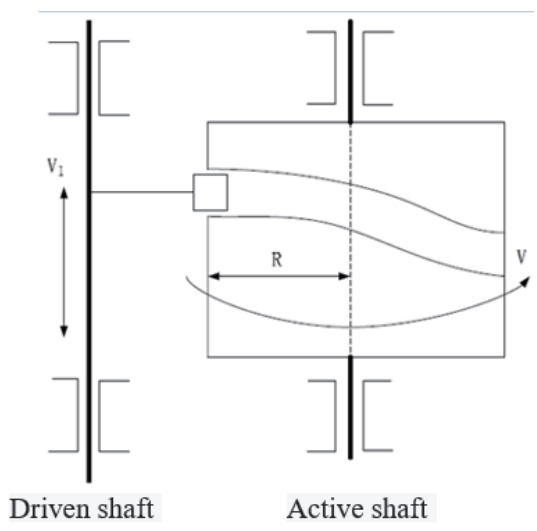

Fig. 4. Schematic diagram of mechanical cam.

A linear arc cam manipulator, a new cam manipulator, and a cam manipulator with adjustable stroke have also been proposed. ${ }^{(16-18)}$ A cam mechanism has a unique output trajectory and must be redesigned when the trajectory needs to be changed, and the output is not flexible. ${ }^{(19)}$ Tu et $a l .{ }^{(20)}$ proposed an ECAM that uses a software and hardware control system and a servo motor to cooperate with the analog cam movement to replace the original physical cam. In this paper, we propose a design for applying ECAMs to a $3 \mathrm{D}$ feeding manipulator. Its servo control system is used in conjunction with a ball screw. We also built the software and hardware interface of the control system. According to the relationship between the motion trajectory of the follower and the coordination of the punch, the output of the manipulator becomes more flexible and intelligent with the help of the cam curve. The flexible mechanical characteristics of the ECAM and the flexible planning of the cam curve are suitable for today's high-precision MC. ECAMs have the ability to index not only the movement of a mechanical cam but also the characteristics of a controllable output movement. In an actual production, a variety of motion trajectories can be visualized by changing the cam curve to improve production efficiency and reduce costs.

\section{System Structure}

We proposed the application of the ECAM synchronization tracking function to a 3D feeding manipulator. To achieve this purpose, a PLC is used as the core of the research. A stamping production line system includes production line equipment, manufacturing process planning, feeder action, a transmission mechanism, and a mechanism protection device. In addition, by considering the product process requirements and equipment layout, a production line equipment requirement table is planned, as shown in Table 1.

The stamping process takes into consideration the issue of on-site moving lines, as shown in Table 2. The process diagram of the workpiece movement is planned on the basis of the sequence of the moving direction of the stamping workpiece and the design of the die, as shown in Fig. 5.

The selection and electrical connection of the hardware involve the punch and 3D feeding manipulator control system and the control system architecture [including the PLC, positioning motion module, IO module, manual pulse generator (MPG), and HMI]. We used a Keynes KV- 
Table 1

Requirements of process equipment.

\begin{tabular}{lccc}
\hline Device name & Specification & Quantity & Function \\
\hline $\begin{array}{l}\text { Punch } \\
\text { 3D feeding } \\
\text { manipulator }\end{array}$ & Bilateral fixture & $1 \mathrm{set}$ & $\begin{array}{c}\text { Material punching and blank stamping formation } \\
\text { to next station for die stamping }\end{array}$ \\
\hline $\begin{array}{l}\text { Two-in-one leveling } \\
\text { machine }\end{array}$ & $\begin{array}{c}\text { Material rack } \\
\text { leveling }\end{array}$ & $1 \mathrm{set}$ & Levels steel coil material \\
\hline $\begin{array}{l}\text { NC left and right } \\
\text { offset feeder }\end{array}$ & Roller type & $1 \mathrm{set}$ & $\begin{array}{c}\text { Length of steel coil material set } \\
\text { according to size of blank }\end{array}$ \\
\hline \begin{tabular}{l} 
Outfeed conveyor \\
\hline
\end{tabular} & Belt type & $1 \mathrm{set}$ & Conveys stamped products to bin \\
\hline
\end{tabular}

Table 2

Stamping process configuration table.

\begin{tabular}{lccc}
\hline & & \multicolumn{2}{c}{ Transfer direction: left $\rightarrow$ right } \\
\hline Stop & Mold & Station & Remarks \\
\hline 1 & Blank forming & Feed & (2) $\begin{array}{c}\text { When the 3D feeding manipulator is transferring, } \\
\text { push blank to 2nd station }\end{array}$ \\
\hline 2 & & 1 & Transfer rough to 3rd station \\
\hline 3 & External molding & 2 & Transfer outer circle forming to 4th station \\
\hline 4 & Inner circle forming & 3 & Move inner circle forming to 5th station \\
\hline 5 & Bottom edge forming & 4 & Move bottom edge forming to 6th station \\
\hline 6 & Punch inner hole & 5 & Move punching to 7th station \\
\hline 7 & Folding forming & 6 & Move products to 8th station \\
\hline 8 & & Discharge & Outfeed conveyor \\
\hline
\end{tabular}

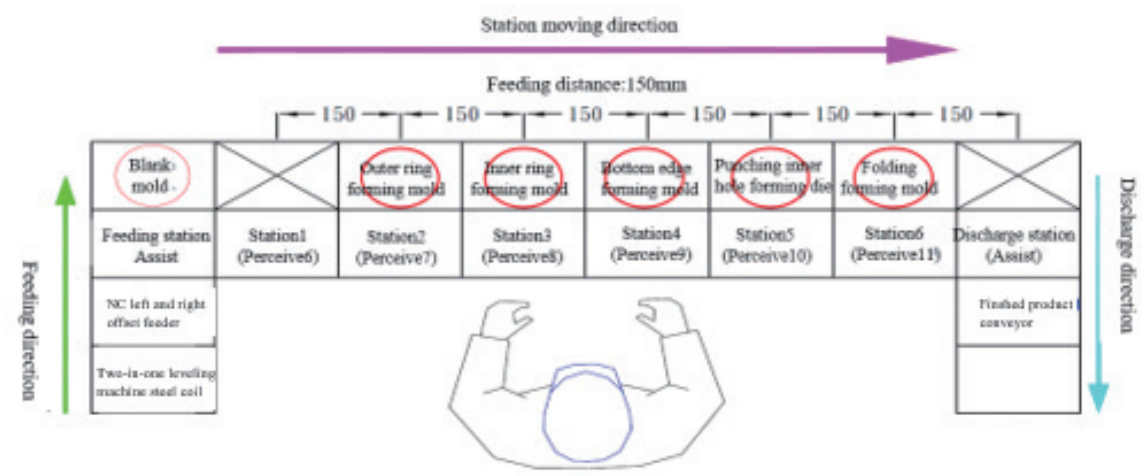

Fig. 5. (Color online) Production line flow.

$7500 \mathrm{CPU}^{(21-23)}$ and a KV-XH16ML motion module. The system uses the MPG to simulate the angle of the crankshaft, and it moved accurately during a trial run of the feeding path with the manual correction of the position. A KV-SSC02 high-speed counter module is mainly used to measure high-speed input signals such as those of sensors and encoders. It provides five counting action modes, three frequency/tachometer action modes, and a combination of counting action and frequency/tachometer action modes. ${ }^{(24)}$ The electrical control system of the 3D 
feeding device includes a controller and a servo motor. The I/O module processes switch signals, such as a fixture detection signal, an axial limit protection sensor signal, an operation switching signal, indicator lights, and handshake signals with the punch. In this study, a Keynes VT5-W10 module was selected. In addition to traditional touch operations, it also supports sliding operations and two-finger zoom in/out. The alarm or graph display can be scrolled intuitively. The communication function includes two Ethernet ports, a VNC client/server function, an FTP server function, a serial connection function, and support for the MODBUS communication protocol. The front is equipped with USB and SD card slots for convenient data transmission and storage.

The transmission motor of the 3D feeding system adopts Yaskawa's seventh-generation $\Sigma 7$ series AC servo unit, which is mainly used in situations that require high speed, high frequency, and high positioning accuracy. This servo unit can maximize the machine performance and minimize the operation time, thus improving production efficiency. ${ }^{(25)}$ The servo motor of our proposed system uses a medium-inertia, high-resolution, 24-bit absolute value serial encoder with 16.77 million pulses/rev. The servo drive unit adopts the Mechatrolink-III motion network, which can improve the performance, precision, and versatility of the automation. The system architecture is shown in Fig. 6. The servo drive unit adopts the Mechatrolink-III motion network, which is shown in Fig. 7.

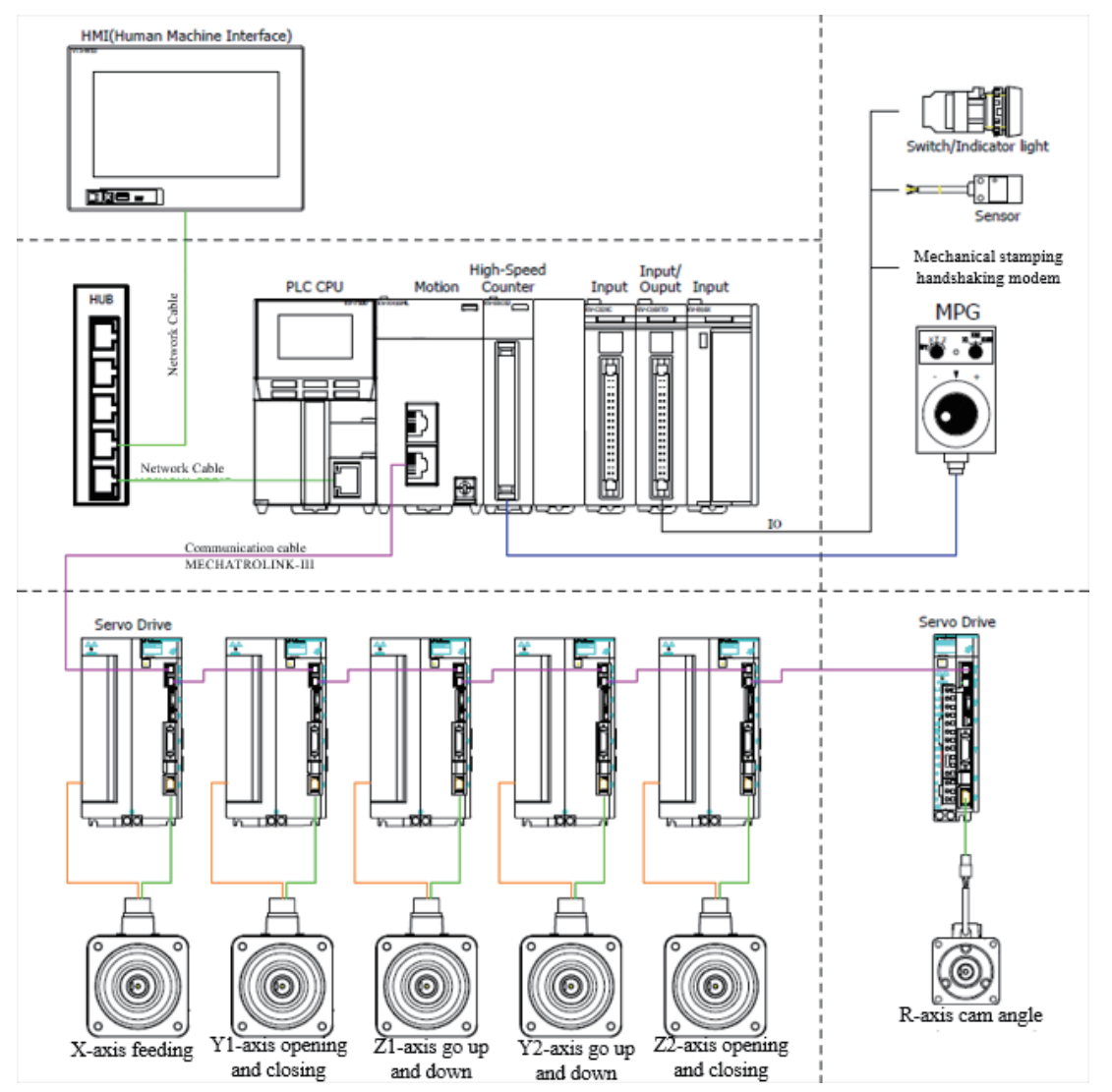

Fig. 6. (Color online) Architecture of control system. 


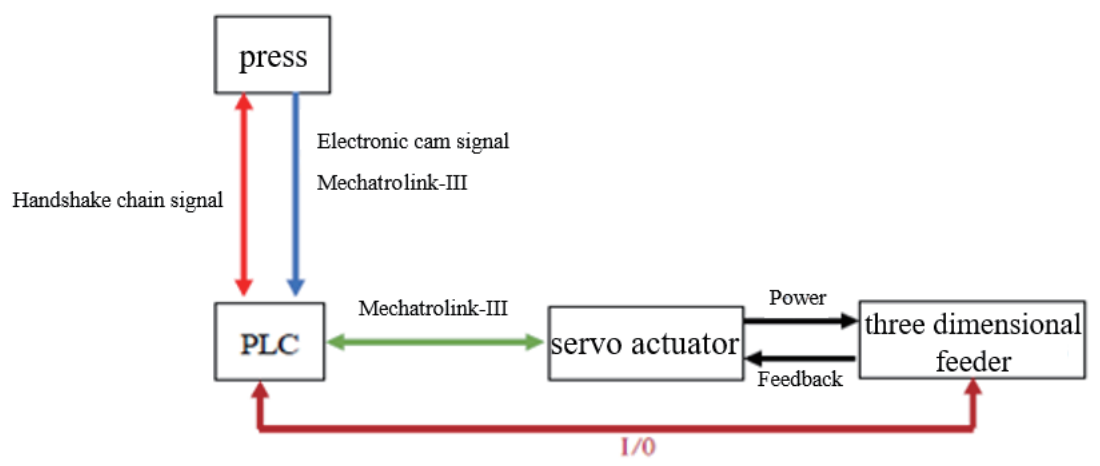

Fig. 7. (Color online) Schematic of system control.

\section{System Testing and Discussion}

In this study, the hardware of the 3D feeder and the assembly of the electronic control system, the punch, and the peripheral equipment are integrated. The PLC program is compiled and the HMI for testing is planned according to the system action requirements. The electronic control systems of the punch and 3D feeder are equipped with a physical handshaking interlock signal and cam angle feedback, so that the actions between the two are coordinated without causing interference, as shown in Fig. 7.

The handshake signals from the punch to the PLC include a punch abnormal signal, a punch stroke signal, a punch linkage signal, a punch origin cam signal, and a punch $70^{\circ}$ cam signal. The handshake signals from the PLC to the punch include "punch operation enable", "punch interlock stop", "punch emergency stop", and "3D feeding manipulator ready to complete".

\section{(1) Calibration}

First, all axial coordinate positions are reset to zero. Before returning the operating position to zero, the corresponding parameters of the mold and the workpiece must be established, as shown in Table 3. The resetting steps are as follows:

Set the mode switch to the calibration mode, and on the HMI menu page, switch the screen to the mechanical parameters to set the axial movement speed and the amount of movement. After setting, switch the screen to the calibration mode screen.

Use the MPG to select axial movement or directly press the axial manual operation switch. Firstly, ensure that the axial and coordinate directions are correct. In case of error, modify the parameters of the motion unit or servo drive. Secondly, move the axis to a position where it does

Table 3

Workpiece parameters.

\begin{tabular}{lc}
\hline Name & Parameter \\
\hline Shift distance (distance between mold and mold center) & $150.0 \mathrm{~mm}$ \\
\hline Clamping distance & $75.0 \mathrm{~mm}$ \\
\hline Lifting height & $10.0 \mathrm{~mm}$ \\
\hline Punching speed (times/minute) & $30-40 \mathrm{SPM}$ \\
\hline
\end{tabular}


not interfere with the punch, operate the punch to stop the slider at the upper starting point $\left(0^{\circ}\right)$, and adjust the mechanism to the original position based on the mold center of the first station for each axis. The $X$-axis is in the backward direction, the $Y$-axis is in the clamping direction, and the $Z$-axis is in the down direction. Thirdly, press the origin return switch for at least $2 \mathrm{~s}$. At this time, the coordinates of all the axes will display 0.000 . The origin indicators of each axis will light up, and the punch origin and 3D origin lamps on the panel will also light up. Lastly, adjust the origin calibration and origin travel protection detection switches of all axes to their appropriate positions. In accordance with the distance between the molds and the diameter and height-related parameters of the workpiece, use the MPG to move each axis to the coordinate to confirm the position, and then adjust the fixture position to ensure that each station can clamp the workpiece and adjust the fixture detected by the sensor. After all the axial positions are confirmed, enter the stroke in the cam setting page. Adjust all the axial positioning, verification and positioning, and travel protection detection switches to their appropriate positions.

\section{(2) Manual operation}

The manual mode has two functions: The first is point-to-point operation and the second is to return to the origin of each axis.

\section{(a) Point-to-point operation}

After mode calibration, the movement strokes in all the axial directions are determined. At this time, we use the axial manual operation switch to make each axis move independently point to point, and use the action of each axis to confirm the accuracy and whether the movements interfere with each other. First, we must understand the action sequence of the 3D feeder, which is shown in Fig. 8.

\section{(b) Return to origin of axes}

During operation, each axis may stop at an indeterminate position. When it is necessary to return to the original position, in addition to using the manual operation switch of the axis, it is also possible to directly press the origin reset button. Each axis then automatically returns to the origin in the order $Y$-axis $\rightarrow Z$-axis $\rightarrow X$-axis.

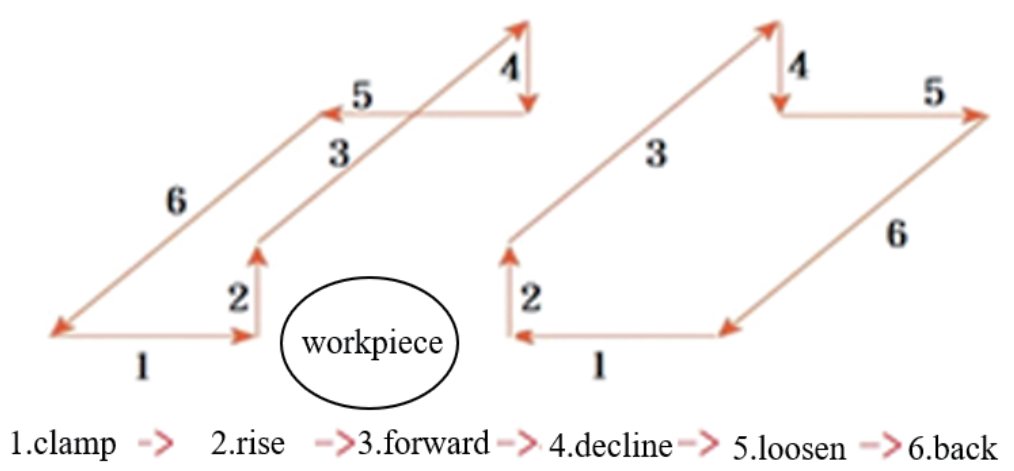

Fig. 8. (Color online) Action sequence diagram of the 3D feeding manipulator, where (1) $Y$-axis advances to clamp the workpiece $\rightarrow Y$-axis moves to forward position $\rightarrow$ (2) $Z$-axis rises to lift the workpiece $\rightarrow Z$-axis rises $\rightarrow$ (3) $X$-axis feeds forward $\rightarrow X$-axis moves forward $\rightarrow$ (4) $Z$-axis moves down to place the workpiece $\rightarrow Z$-axis moves down to position $\rightarrow(5) Y$-axis retreats to release the workpiece $\rightarrow$ (6) $X$-axis retreats to the standby position $\rightarrow$ $X$-axis retreats backward to complete a cycle of action $\rightarrow$ punch test press. 


\section{(3) Handwheel simulation operation}

After manual operation and repeated testing, it is determined that there is no interference between the position, the axial direction, and the mold during movement, and the workpiece can be accurately transferred to the mold at the next station. The next step is to plan the cam path to achieve the synchronous tracking action. (a) First, pre-plan the angle of cooperation between the $X / Y / Z$-axis and the punch press. Table 4 gives the allocation of action angles. (b) Enter the cam setting selection screen to select the axial setting. Taking the $X$-axis as an example, the setting steps are as follows: enter the cam data, set instructions, use the axial number and cam data, and set the number of segments. Finally, read the preset cam curve.

\section{(4) Linked operation}

After the handwheel simulation, the correlation of the three-axis motion path trajectory and the angle is confirmed. The next step is to physically match the punching action. However, before that, we can use the continuous test mode (idle running) function to perform another confirmation test. At the end of the operation steps, the punch is pressed to stop it, then the punch stops at the upper starting point, the feeding manipulator stops, the continuous test (idle running) function is completed, and subsequently this function is closed. The operation steps are as follows.

(1) Adjust the punch slider to the normal punching position and set the punch operating mode to a safe stroke.

(2) Switch the HMI to the mechanical parameter page, switch the feed-following function to the feed-stroke mode, and set the speed of the virtual axis. Consequently, the ECAM spindle input will switch to the virtual spindle.

(3) Initially, there is no workpiece in the mold, so first go to the fixture setting screen to turn off the fixture inspection.

(4) Press the punch start to make the punch press once, and the punch will stop at the upper starting point. Concurrently, the punch origin cam signal will notify the virtual axis to start.

(5) The virtual axis starts to rotate to generate an angle, and the synchronous follow-up action of each axis will be the same as the cam angle of the R-axis. The feeding manipulator will move the workpiece to the next station according to the cam curve track and return to the standby position.

(6) Repeat step (4) until all molds have workpieces.

Table 4

Distribution of axial movement angles.

\begin{tabular}{lcc}
\hline Action & Starting angle (deg) & End angle (deg) \\
\hline Clamp & $255^{\circ}$ & $310^{\circ}$ \\
\hline Rise & $315^{\circ}$ & $335^{\circ}$ \\
\hline Go ahead & $338^{\circ}$ & $25^{\circ}$ \\
\hline Decline & $30^{\circ}$ & $50^{\circ}$ \\
\hline Release & $55^{\circ}$ & $90^{\circ}$ \\
\hline Back & $90^{\circ}$ & $210^{\circ}$ \\
\hline
\end{tabular}


(7) When the size of the stamping workpiece is stable, switch the feed-stroke mode to the feedfollowing mode. Subsequently, the ECAM spindle input will be switched to the R-axis, then the punching operation mode will be switched to interlock. Following that, the fixture detection switch function will be automatically turned on to prevent situations where the operator forgets to turn it on and damages the mold and equipment.

(8) Press the punch to start. The feeding manipulator continues to follow up with the action synchronously.

(9) Slowly increase the punching speed of the punch to reach the highest production capacity.

(10) In formal stamping production, it is necessary to correctly set the start and end angles of the fixture detection. Within this detection angle range, if any detector fails to detect the angle, the punch will be instructed to perform an emergency stop, enabling the feeding manipulator to stop and release the synchronous tracking function.

(11) When the abnormality is eliminated, the feed-stroke function can be used again to test stamping, and normal production can be restored after confirming that the action is normal.

Figure 9 shows the operation monitoring display screen during continuous production. It shows the punch stroke per minute (SPM), the current angle of the main shaft, the current position of the slave axis, the stroke setting, the synchronization of the starting angle, the fixture detection indicator, the punch and slave axis origin indicator, the drive status, and any abnormal information.

When an anomaly occurs, we can switch to the anomaly history screen, display the message informing of the abnormality, record the number and time of the anomaly, confirm it, and delete the record. We can also enter the PLC IO monitoring screen to monitor signals for preliminary fault diagnosis.

During the test on the feeding manipulator, several issues need to be discussed, including fixture crossbar and mold design issues.

\begin{tabular}{|c|c|c|c|c|c|c|c|}
\hline & \multicolumn{2}{|c|}{$2020-10-31$} & \multicolumn{2}{|c|}{ Operation monitoring } & & \multicolumn{2}{|l|}{$10: 41: 59$} \\
\hline & & $\begin{array}{c}\text { detec } \\
06\end{array}$ & $\begin{array}{l}\text { detect } \\
07\end{array}$ & $\begin{array}{c}\text { detect } \\
08\end{array}$ & $\begin{array}{c}\text { detect } \\
09\end{array}$ & $\begin{array}{c}\text { detect } \\
10\end{array}$ & $\begin{array}{c}\text { detect } \\
11\end{array}$ \\
\hline press SPN & 0 & press origin & $\begin{array}{c}\mathrm{X} \text {-axis } \\
\text { origin }\end{array}$ & $\begin{array}{l}\text { Y1-axis } \\
\text { origin }\end{array}$ & $\begin{array}{l}\text { Z1-axis } \\
\text { Origin }\end{array}$ & $\begin{array}{l}\text { Y2-axis } \\
\text { origin }\end{array}$ & $\begin{array}{c}\mathrm{Z2} \text {-axis } \\
\text { origin }\end{array}$ \\
\hline axial & current position & stroke setting & sync setting & sync angle & error code & warning code & diver \\
\hline $\mathrm{R}$-axis & 358.496 & & 0 & 0 & 0 & 0 & 0 \\
\hline $\mathrm{X}$-axis & 0.000 & 0 & 0 & 0 & 0 & 0 & 0 \\
\hline Y1-axis & 0.000 & 0 & 0 & 0 & 0 & 0 & 0 \\
\hline Y2-axis & 0.000 & 0 & 0 & 0 & 0 & 0 & 0 \\
\hline Z1-axis & 0.000 & 0 & 0 & 0 & 0 & 0 & 0 \\
\hline Z2-axis & 0.000 & 0 & 0 & 0 & 0 & 0 & 0 \\
\hline
\end{tabular}

Fig. 9. Monitoring of HMI operation. 
(1) There is an error in the reference plane of the clamp crossbar during processing, making the two crossbars unparallel after installation and making it impossible to clamp the workpiece at the two stations when the clamp has advanced and the workpiece is clamped. This issue is eliminated after reprocessing.

(2) There is an issue with the mold design. When the punch is at the upper starting point, the space between the upper and lower molds is insufficient, causing the fixture to wait until the cam angle of the punch is $255^{\circ}$ before it can advance and clamp the workpiece, and the fixture stroke is $75.5 \mathrm{~mm}$, which affects other steps of the operation. The starting angle of the axial synchronization must be increased and the action angle range must be minimized to achieve a faster axial movement and higher inertia. However, this results in the insufficient strength of the fixture crossbar and vibration, affecting the stability of the fixture and resulting in the workpiece being incorrectly clamped. Thus, fixture detection anomalies often occur. To solve this problem, we can only sacrifice production capacity by reducing it from 40 SPM in the original design to 33 SPM.

(3) Through the real-time monitoring of the sequence diagram and the analysis of the current coordinates, Y1, Y2, Z1, and Z2 are well synchronized, as shown in Fig. 9. According to the current and command coordinates used to analyze the synchronous tracking, the $X$-axis is about $4.07 \mathrm{~mm}$ behind the command coordinate, the $Y$-axis is behind by about $1.33 \mathrm{~mm}$, and the $Z$-axis is behind by about $1.08 \mathrm{~mm}$, indicating that the synchronous tracking is satisfactory. The lag can be corrected by correcting the tracking error through adjusting the rigidity, response, or cam phase of the servo unit.

\section{Conclusions}

We developed a PLC ECAM synchronous tracking function through the coordination of a 3D feeding manipulator and the movement trajectory of a punching machine with the aim of improving stamping productivity and feeding accuracy. The PLC control system of the feeder is connected and coordinated with a Yaskawa $\Sigma 7$ series AC servo unit based on Mechatrolink-III communication. According to the results of production tests on site, the overall functionality meets the requirements in terms of accuracy, speed, synchronization, and immediacy. Moreover, the cam data can be obtained directly through the HMI and can be modified in real time without stopping the machine, thus demonstrating its simplicity and convenience and reducing the production time. The feeder was installed in a production line. Without the proposed 3D feeder, the production line with five operators working $8 \mathrm{~h}$ a day had an output of about 3000 pieces. With the 3D feeder, a stamping speed of 33 SPM, and one operator working $8 \mathrm{~h}$ a day, the output was about 15000-16000 pieces. The production capacity was thus increased more than fivefold and the labor and equipment costs were reduced. Owing to the increased production capacity, greater punctuality, and faster delivery of orders, the number of orders received by this company increased and the return on investment was high. 


\section{Acknowledgments}

This research work was partly supported by the Ministry of Science and Technology, R.O.C., Grant No. MOST 110-2221-E-992-093.

\section{References}

1 Y. J. Chen: Motion Simulation and Analysis of Stamping Roller Feeding Mechanism, Master's Thesis (Department of Die \& Mold Engineering, National Kaohsiung University of Applied Science and Technology, Taiwan, R.O.C., 2015).

2 J. H. Zhou: Special Study on the Technology Roadmap of Metal Secondary Processing-Stamping and Forging (Metal Center, Taiwan 2003) 1st ed., Chaps. 3 and 4.

3 O. S. Wen: Research and Development of a Kind of Automatic Loading and Unloading Manipulators, Master's Thesis (Hefei University of Technology, 2018).

4 Shungdar Industrial Co., Ltd.: https://www.shungdar.com.tw/zh-TW/index/index.html (accessed June 2020).

5 Kunfeng Metal Industry Co., Ltd.: https://www.twincn.com/item.aspx?no=22665661 (accessed June 2021).

6 W. Huang: Design of Three-dimensional Feeding Manipulator and Planning and Optimization of Trajectory, Master's Thesis (Northeastern University, 2015).

7 L. Sun: Development and Research on the Manipulator for Loading and Unloading of Stamping Machines, Master's Thesis (South China University of Technology, 2015).

8 F. Wu: Research on Coordinated Control of Feeding Manipulator and Servo Press, Master's Thesis (Hubei University of Technology, 2017).

9 Y. J. Wang and H. Ding: Forg Technol. 41 (2016) 55. https://doi.org/10.13330/j.issn.1000-3940.2016.06.011

10 X. L. Zhou: Research and Optimal Design of Three-coordinate Transmission Mechanism of Multi-feed Press, Master's Thesis (Yanshan University, 2017).

11 Keynes: Positioning/Motion Control Unit KV-XH16ML/KV-XH04ML User Manual. (Keynes, Taiwan, 2019) 2nd ed.

12 J. S. Liang and J. J. Cao: Light Ind. Mach. 4 (2004) 108. https://www.ixueshu.com/ document/1ac2992c2edbc0ac51db128870157231318947a18e7f9386.html

13 M. I. Fukuoka: United States Patent (US6781339B1) (2004) 1. https://patents.google.com/patent/US6781339/zh

14 Z. H. Ge, J. J. Shen, and X. Y. Si: Food Mach. 33 (2017) 73. http://ifoodmm.com/ uploadfile/2017/1031/20171031050237330.pdf

15 X. X. Luo and Y. He: Mech. Eng. 1 (2019) 44. http://big5.oversea.cnki.net/kcms/detail/detail. aspx?filename $=$ JXGU201901013 $\&$ DBName $=$ cjfqtotal $\&$ dbcode $=$ cjfq\&uid $=$ WEEvREcwSlJHSldSdmVqeVpQU 2FpY3FWNzJ1eGtMemplTW0xNFJGRU5Baz0=\$9A4hF YAuvQ5obgVAqNKPCYcEjKensW4IQMovwHtwkF 4VYPoHbKxJw!!

16 L. Chang: Manuf. Autom. 33 (2010) 155. https://wenku.baidu.com/view/cfa82022482fb4daa58d4bf6.html

17 W. Yuan: Light Ind. Mach. 6 (2012) 1. https://big5.jinyueya.com/magazine/31584439.htm

18 J. Chu: Mechanical Design and Practical Mechanism Motion Simulation Diagram (Electronic Industry Press, China, 2020) 3rd ed..

19 X. Wang: Small Internal Combust. Engine Vehicle Tech. 46 (2017) 47. http://222.198.130.40:81/Qikan/Article/ Detail?id $=673702914$

20 X. Tu, J. Zou, and J. Zhang: Micro Special Motor 43 (2015) 70. https://wenku.baidu.com/ view/412bcc60777f5acfalc7aa00b52acfc789eb9f3d.html?re=view

21 Keynes: User Manual of Programmable Controller KV-7000 Series (Keynes, Taiwan, 2018) 2nd ed.

22 Keynes: KV-7000 Series Catalog of Programmable Logic Controllers (Keynes, Taiwan, 2019) 2nd ed.

23 Keynes: Comprehensive Catalog of Positioning/Sports (Keynes, Taiwan, 2018) 2nd ed.

24 Keynes: High-Speed Counter Module KV-SSC02 User Manual (Keynes, Taiwan, 2018) 4th ed.

25 YASKAWA: $\Sigma$-7S Servo Unit MECHATROLINK-III Communication Instruction Type Product Manual (YASKAWA, Taiwan, 2015) 1st ed.

26 YASKAWA: https://www.yaskawa.com.tw/ (accessed June 2021).

27 L. Nan Leiying, C. Qi, and Y. Sun: Forg. Equip. Manuf. Technol. 41 (2006) 18. https://www.zip118. com/205196481_0203/49020.html 